\title{
Electric field effects on CNTs/vinyl ester suspensions and the resulting electrical and thermal composite properties
}

\author{
Hilmi Yurdakul ${ }^{a}$, A. Tugrul Seyhan ${ }^{\mathrm{a}, *}$, Servet Turan ${ }^{\mathrm{a}}$, Metin Tanoğlu ${ }^{\mathrm{b}}$, Wolfgang Bauhofer ${ }^{\mathrm{c}}$, Karl Schulte ${ }^{\mathrm{d}}$ \\ ${ }^{a}$ Department of Materials Science and Engineering, Anadolu University (AU), Iki Eylul Campus, 26550 Eskisehir, Turkey \\ ${ }^{\mathrm{b}}$ Mechanical Engineering Department, Izmir Institute of Technology (IZTECH), Gulbahce Koyu 35437, Izmir, Turkey \\ ${ }^{\mathrm{c}}$ Department of Materials in Electrical Engineering and Optics Section, Technicshe Universitat Hamburg-Harburg (TUHH), D-21073 Hamburg, Germany \\ ${ }^{\mathrm{d}}$ Polymer Composites Section, Technicshe Universitat Hamburg-Harburg (TUHH), D-21073 Hamburg, Germany
}

\section{A R T I C L E I N F O}

\section{Article history:}

Received 15 June 2010

Accepted 12 August 2010

Available online 18 August 2010

\section{Keywords:}

A. Polymer-matrix composites (PMCs)

A. Carbon nanotubes

B. Electrical properties

C. Anisotropy

D. Transmission electron microscopy (TEM)

\begin{abstract}
A B S T R A C T
In this study, electrical conductivity of a vinyl ester based composite containing low content $(0.05,0.1$ and 0.3 wt.\%) of double and multi-walled carbon nanotubes with and without amine functional groups (DWCNTs, MWCNTs, DWCNT- $\mathrm{NH}_{2}$ and MWCNT- $\mathrm{NH}_{2}$ ) was investigated. The composite with pristine MWCNTs was found to exhibit the highest electrical conductivity. Experiments aimed to induce an aligned conductive network with application of an alternating current (AC) electric field during cure were carried out on the resin suspensions with MWCNTs. Formation of electric anisotropy within the composite was verified. Light microscopy (LM), scanning electron (SEM) and transmission electron microscopy (TEM) were conducted to visualize dispersion state and the extent of alignment of MWCNTs within the polymer cured with and without application of the electric field. To gain a better understanding of electric field induced effects, glass transition temperature $\left(T_{\mathrm{g}}\right)$ of the composites was measured via Differential Scanning Calorimetry (DSC). It was determined that at $0.05 \mathrm{wt} . \%$ loading rate of MWCNTs, the composites, cured with application of the AC electric field, possessed a higher $T_{\mathrm{g}}$ than the composites cured without application of the AC electric field.
\end{abstract}

(c) 2010 Elsevier Ltd. All rights reserved.

\section{Introduction}

Polymers are currently modified with nano-sized additives to develop advanced multi-functional composites. When added to polymers at very low concentrations, CNTs are perfectly capable of forming a conductive network due to their extraordinary high conductivity and large aspect ratio [1-7]. In addition, they have outstanding mechanical and thermal properties. Therefore, CNTs are considered the most promising reinforcing constituent to manufacture electrically conductive polymer composites with at least retained or improved mechanical properties [2-4]. In general, polymer composites used for electronic, automotive and aerospace applications are required to dissipate static electric loads to avoid electrostatic charging and electromagnetic radio frequency interference. Carbon black (CB) is commonly used as a conductive filler for this purpose. CNTs with aspect ratio several orders of magnitude higher than that of $\mathrm{CB}$ are expected to make polymers conductive at relatively low concentrations. A low content of fillers can provide polymer composites with ease of processing accompanied by surface smoothness and enhanced mechanical properties.

\footnotetext{
* Corresponding author. Fax: +90 2223213575.

E-mail address: atseyhan@anadolu.edu.tr (A.T. Seyhan).
}

It was determined that the replacement of $\mathrm{CB}$ with $\mathrm{CNTs}$ reduced the concentration required for electrical conductivity from 5 to $0.04 \mathrm{wt} . \%$ and increased the overall conductivity of polymer composites [2,3]. Therefore, the use of CNTs instead of CB seems a very feasible approach to achieving conductive polymer composites without any mechanical property degradation [2-8].

The electrical conductivity of composites is mainly based upon percolated pathways of conductive particles. The percolation theory is used to account for the conductivity of polymer composites [5-10]. In this theory, the percolation threshold refers to the onset of electrical conductivity due to conductive pathways formed once a critical filler concentration is accomplished in a given polymer system. Nevertheless, the percolation theory was in fact established for spherical particles. Therefore, it shows relatively poor validity for CNTs with very large aspect ratio $(l / d \gg 1)$ compared to spherical-like fillers. An advanced percolation theory taking into account the aspect ratio of fillers was proposed to predict the critical volume fraction at which a percolated network is formed with conductive fillers [5-9]. Accordingly, critical volume contents for percolation threshold of rod-like fillers with an aspect ratio of more than 100 were predicted and varied from 0.24 to 1.35 vol.\% [3-5]. However, even in this case, experimentally obtained percolation threshold values were found to be far below the theoretical predictions because their theoretical approach is no longer associ- 
ated with either the Brownian motion of the fillers, or with any cohesive forces including particle-particle or particle-matrix interactions.

Process induced negative surface charges on $\mathrm{CB}$, carbon nanofibers (CNFs) and CNTs may be utilized to produce initially charged stabilized dispersions, which become then capable of forming aligned networks under application of either direct current (DC) or alternating current $(A C)$ electric fields $[8,9]$. In principle, the resulting structure of the conductive filler networks is highly dependent on the type of electric field applied during cure. Prasse et al. [8] applied an AC electric field to induce the alignment and to percolate a conductive network of CNFs within an epoxy resin suspension during cure. They found that the maximum composite conductivity induced by CNFs is not significantly larger than that induced by $C B$ within the same epoxy matrix resin. Martin et al. $[10,11]$ concluded that both AC and DC electric fields can be utilized to induce the formation of aligned CNT networks by adjusting the gap between electrodes being in contact with the dispersion. Moreover, they also stated that more uniform and more aligned networks can be accomplished in an AC electric field than in a DC electric field because the DC electric field leads to relatively inhomogeneous and branching network structures.

SEM allows the examination of conducting filler particles in an insulating polymer matrix. Detection of selected secondary electrons is required to resolve nano sized filler particles. However, every SEM detector is not capable of monitoring the small changes of the morphology of the polymer matrix induced by the conducting filler particles. Kovacs et al. [12] studied the influence of SEM parameters and the challenges associated with the accurate image interpretation from MWCNT filled epoxy composites. They acquired the SEM pictures of MWCNTs by exploiting the charge contrast. They concluded that all monitored electrons seem to be emitted within approximately $50 \mathrm{~nm}$ of the sample depth, and that no reliable information is further accessible from deeper regions of the sample, even that the acceleration voltage was increased. A similar study was performed by Loos et al. [13]. They visualized the dispersion of SWCNTs within polystyrene by charge contrast SEM and bright field TEM techniques. They concluded that the number of visible SWCNTs in the polymer matrix increases with increasing energy of the primary beam. They also showed that with the charge contrast imaging technique it is possible to obtain information about the mechanisms to form three dimensional conductive networks of CNTs within the matrix polymer.

This study firstly focuses on the evaluation of electrical conductivity of a vinyl ester based polymer composites containing low content of MWCNTs, DWCNTs, MWCNT-NH$H_{2}$ and DWCNT-NH $\mathrm{H}_{2}$. Subsequently, using advanced microscopy techniques, it was intended to pinpoint AC electric field induced effects on the resulting electrical and thermal composite properties.

\section{Experimental}

\subsection{Materials}

Thin multi-walled carbon nanotubes (MWCNTs) and double walled carbon nanotubes (DWCNTs) with and without amine functional $\left(\mathrm{NH}_{2}\right)$ groups were purchased from Nanocyl (Namur, Belgium) and used as reinforcing constituents for the matrix resin. A specially synthesized styrene-free polyester resin POLIYA 420 and a commercial vinyl ester-epoxy resin (bisphenol A epoxy based) POLIPOL 701 with 35 wt.\% of styrene were obtained from POLIYA Polyester, Turkey. The formulized resin blend was composed of $25 \mathrm{wt} . \%$ of POLIYA 420 and $75 \mathrm{wt} . \%$ of POLIVES 701. Styrene emission agent BKY 740, from Alton Chemie, Germany, was used to prevent evaporation of styrene from the resin during the dispersion process. To polymerize the resin suspensions, Cobalt naphthenate (CoNAP) and methyl ethyl ketone peroxide (MEKP) were used as accelerator and initiator, respectively.

\subsection{Preparation of composites with CNTs}

A 3-roll mill (Exact $120 \mathrm{~S} \mathrm{GmbH}$ ) was employed to perform proper dispersion of the CNTs within the matrix resin at various loading rates $(0.05,0.1$ and $0.3 \mathrm{wt} . \%)$ under intensive shear. This technique was successfully conducted for epoxy/CNTs systems [14]. The prepared suspensions were allowed to cure at room temperature followed by post-curing at $120^{\circ} \mathrm{C}$ for $2 \mathrm{~h}$.

\subsection{Alignment of CNTs induced by AC electric field}

To induce alignment of the CNTs within the polymer matrix, resin suspensions with $0.005,0.02$ and 0.05 wt.\% of MWCNTs were exposed to a sinusoidal AC electric field of $400 \mathrm{~V} / \mathrm{cm}$ during cure by means of parallel brass plates. Following the cure of the resin suspensions with application of the AC electric field, the resulting composites were subjected to post-curing at $120^{\circ} \mathrm{C}$ for $2 \mathrm{~h}$. The samples were subsequently cut and contacted in the directions parallel and perpendicular to the direction of the AC electric field applied to assess the anisotropy of electrical conductivity in the composites. The given field strength refers to the peak value of the AC electric field which was applied at a frequency of $1 \mathrm{kHz}$ throughout the experiments. Note that various sinusoidal AC electric field values above $400 \mathrm{~V} / \mathrm{cm}$ were also applied during cure in order to induce more alignment of CNTs within the resultant polymer. However, surfaces of the brass plates in contact with the resin suspensions were overheated due to high electric field applied during cure and an excessive exothermic reaction eventually occurred, with the resin samples getting burnt. On the other hand, no electric anisotropy was observed when the electric field values were kept lower than $400 \mathrm{~V} / \mathrm{cm}$ during cure of the resin suspensions.

\subsection{Electrical conductivity measurements}

Electrical conductivity of the CNT modified composites cured with and without application of the AC electric field was measured by dielectric spectroscopy using a HP 4284a impedance analyzer. For each set, at least three specimens with a dimension of $10 \times 10 \times 1 \mathrm{~mm}$. were tested with voltage amplitude of $1.0 \mathrm{~V}$ in a frequency range between $20 \mathrm{~Hz}$ and $1 \mathrm{MHz}$. The corresponding electrical conductivity of the samples was calculated from the complex impedance $\left|Z^{*}\right|$ with respect to the frequency according to the equation given below.

$\sigma(v)=1 /\left|Z^{*}(v)\right| t / A$

where $\sigma$ is the electrical conductivity, $v$ is the frequency, $t$ is the thickness of the sample, and $A$ is the cross sectional area of the specimens.

\subsection{Microscopic examinations}

Light microscope (Olympus BH2), and Schottky emitter field emission gun (FEG) scanning electron microscope (SEM-Zeiss SUPRA $50 \mathrm{VP}$ ) equipped with In-Lens and QBS detectors in variable pressure (VP) mode at $30 \mathrm{kV}$ were conducted to visualize the extent of alignment of CNT networks on the fracture surfaces of the samples which were cut in liquid nitrogen with a razor blade parallel to the AC electric field applied. To avoid charging, silver particles were doped onto surfaces of the samples prior to SEM examination. TEM sample films with a thickness of $70 \mathrm{~nm}$ were prepared at room temperature by using an ultra-microtome. TEM studies were performed by using $200 \mathrm{kV}$ acceleration voltages with 
a Schottky emitter field emission gun (FEG) transmission electron microscope (TEM-Jeol2100F) coupled with a scanning transmission electron microscope high angle annular dark field detector (STEM/HAADF-Fishione) and energy dispersive X-ray spectrometer (EDX-JED2300T). The probe size with a diameter of $1 \mathrm{~nm}$ was employed to enhance the resolution during EDX elemental analysis.

\subsection{DSC measurements}

A TA Instrument Q-10 DSC was used to measure $T_{\mathrm{g}}$ of the composites cured with and without application of an AC electric field. A small quantity of the samples (5-10 mg) was used for the measurements. Runs were carried out, using an aluminum empty pan as a reference. Each sample was first heated from room temperature up to $200{ }^{\circ} \mathrm{C}$ with a constant heating rate of $5{ }^{\circ} \mathrm{C} / \mathrm{min}$ in nitrogen atmosphere. Once cooled down to room temperature, the same samples were reheated up to $200{ }^{\circ} \mathrm{C}$ with a heating rate of $5{ }^{\circ} \mathrm{C} / \mathrm{min}$ to determine the $T_{\mathrm{g}}$ values.

\section{Results and discussion}

Fig. 1 shows the DC electrical conductivity of the amino-functionalized and non-functionalized DWCNT and MWCNT loaded vinyl ester cured without application of an AC electric field as a function of weight content of the CNTs. The percolation threshold, which is defined as the filler content to achieve a conductivity of $10^{-6} \mathrm{~S} / \mathrm{m}$, is hypothetically accepted to be lower for fiber-shaped fillers than for spherical ones [8]. As seen in the figure, regardless the type of CNTs or weight content, composites with untreated CNTs exhibit higher electrical conductivity values than those with amino-functionalized CNTs. The lowest percolation threshold value, which is below 0.05 wt.\%, was observed for MWCNTs. Moreover, the percolation threshold value of DWCNTs was found to be in the range of 0.05 and $0.1 \mathrm{wt} . \%$. On the other hand, amino-functionalized CNTs showed a percolation threshold at much higher loading rates than given in this study. Amino functional groups grafted onto the CNT surface disturbed the graphitic structure of CNTs and reduced the conductivity of the tubes itself by forming an electrically insulating thin layer on the outer surface of CNTs that is highly compatible with the surrounding polymer matrix re-

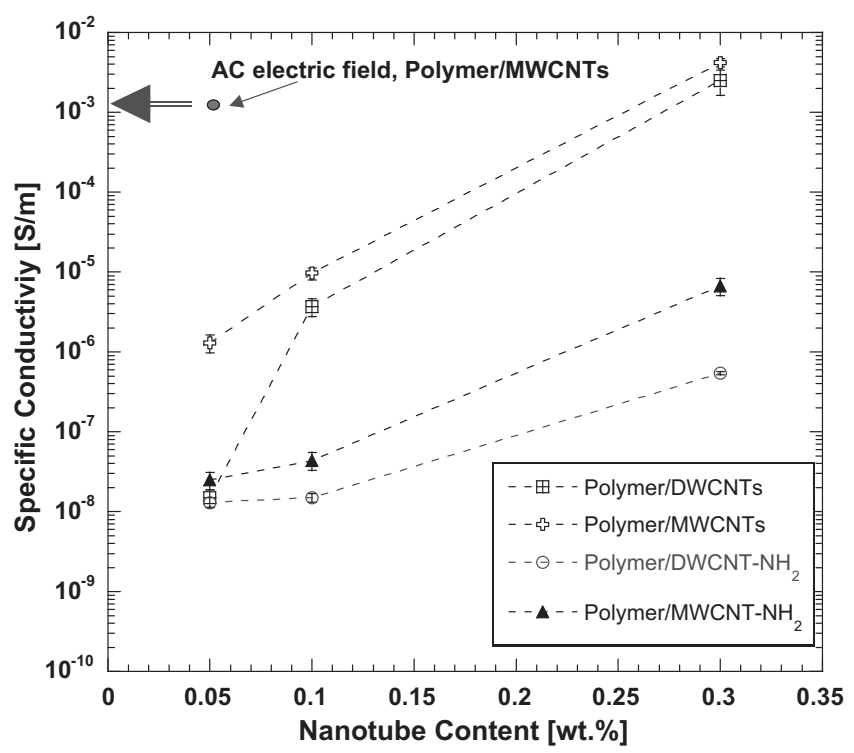

Fig. 1. DC conductivity of functionalized and non-functionalized DWCNT and MWCNT loaded vinyl ester cured without application of AC electric field as a function of weight content of the CNTs. sin and leads to a higher tunneling distance between CNTs. In addition, the CNTs are ruptured and their aspect ratio is lowered during chemical functionalization via ball milling. Based on the information provided by the manufacturer of the CNTs, the aspect ratio of MWCNT-NH $\mathrm{N}_{2}$ is five times lower than that of MWCNTs, while, on the other hand, the DWCNTs have an aspect ratio twice as high as that of DWCNT- $\mathrm{NH}_{2}$. As a result, the percolation threshold, which is highly related to the aspect ratio, shifted to higher values for amino-functionalized CNTs. Note that this is indeed not a new finding, as similar studies were already reported in the literature on epoxy/CNT composites [2,3].

Based on the results obtained, at each loading rate, MWCNTs resulted in the highest electrical conductivity in the polymer, as shown in Fig. 1. Therefore, MWCNTs were reasonably selected as filler constituents in the further experiments aimed to align CNTs in the resin suspension with the application of AC electric field during cure. Fig. 2 shows the typical specific electrical conductivity of the composites cured with application of the AC electric field with respect to frequency on a log-log scale in the directions parallel and perpendicular to the electric field applied. Note that the composites containing $0.005 \mathrm{wt}$.\% of MWCNTs show a typical dielectric behavior like neat polymer such that its electrical conductivity steadily increases on the log-log scale. Samples with $0.05 \mathrm{wt} . \%$ of MWCNTs exposed to the given AC electric field showed a frequency independent electrical conductivity. On the other hand, samples with $0.02 \mathrm{wt}$ \% MWCNTs have a similar response up to a specific knee frequency above which a transition to dielectric behavior is observed. Furthermore, composites with $0.02 \mathrm{wt} . \%$ MWCNTs were found to exhibit almost an order of magnitude higher conductivity parallel to the AC electric field than perpendicular. Similarly, composites containing $0.05 \mathrm{wt} \%$ of MWCNTs showed a bit higher conductivity parallel to the AC electric field than perpendicular. Note that it is virtually impossible to achieve the same degree of AC electric field induced CNT alignment in all points of the composite. That is why electric anisotropy was not detected in all areas of the samples sectioned from the same composite. Moreover, at $0.05 \mathrm{wt} . \%$ loading rate of MWCNTs, conductivity of the composites cured without application of the AC electric field is about three orders of magnitude lower than conductivity of the composites $\left(10^{-6} \mathrm{~S} / \mathrm{m}\right.$ as seen in Fig. 1$)$ cured with the appli-

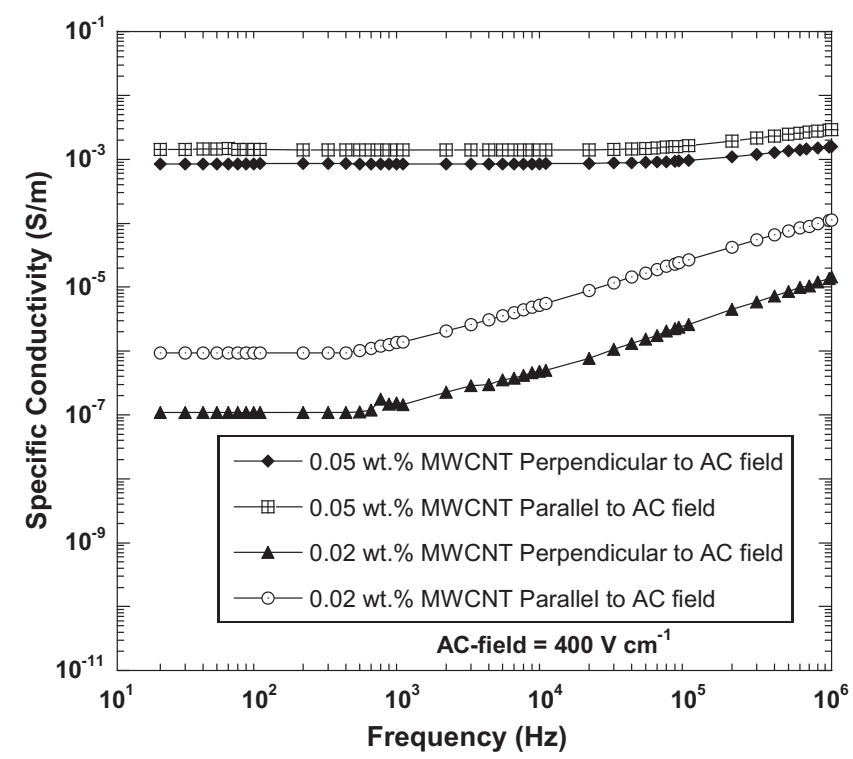

Fig. 2. Typical resulting specific conductivity of the composites cured with application of AC electric field with respect to frequency in the directions parallel and perpendicular to the AC electric field. 
cation of the AC electric field $\left(10^{-3} \mathrm{~S} / \mathrm{m}\right.$ indicated by a gray circle in Fig. 1). This implies that the AC electric field applied during cure alter the orientation, size, array and growth of CNT agglomerates to some extent, which in turn reduces the apparent percolation threshold value of the resultant composites.

Fig. $3 \mathrm{a}$ and $\mathrm{b}$ shows the light micrographs of thin polished film samples sectioned from 0.05 wt.\% of MWCNTs containing composites cured with and without application of the AC electric field, respectively. Note that there is a slight variation in thickness of the samples due to polishing, which leads to a change in contrast in micrographs. As seen in Fig. 3a, uniform and slightly aligned structures as well as relatively large clusters indicated by the white arrows were observed in the composites cured with application of the AC electric field. On the other hand, relatively large and more CNT agglomerates with inhomogeneous distribution are visible within the composites cured without application of the AC electric field, as depicted in Fig. 3b. Martin et al. [10] pointed out that polarization of CNTs in an electric field leads to an additional attractive interaction between individual CNTs and the existing

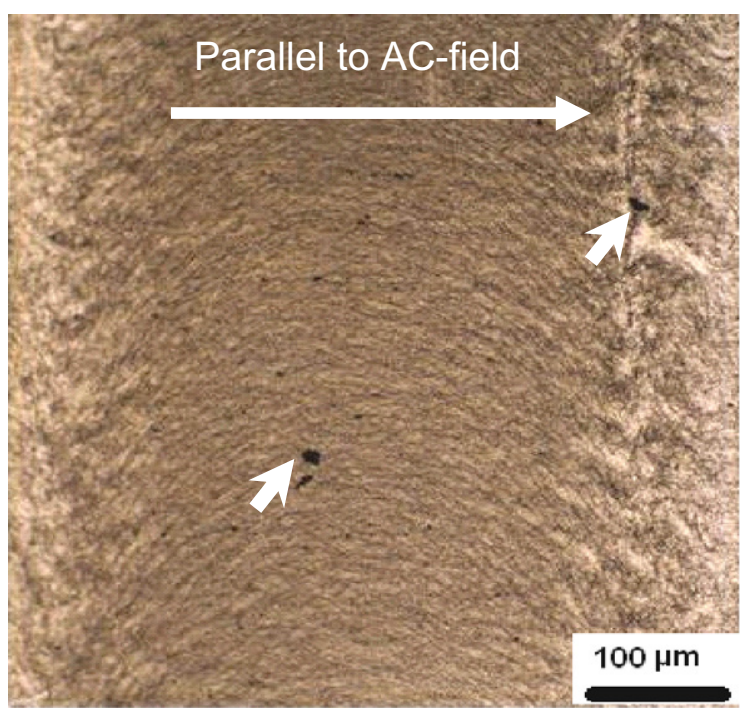

(a)

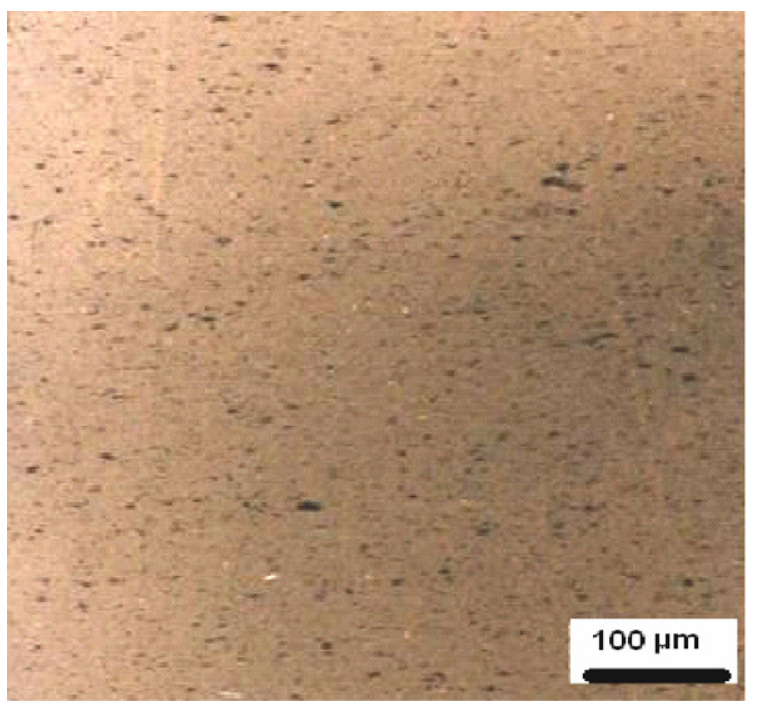

(b)

Fig. 3. Light micrographs of the polished thin film samples sectioned from the composites with 0.05 wt.\% of MWCNTs, cured with (a) and without (b) application of the AC electric field.
CNTs bundles, which is of great importance in characteristics of conductive networks formed within the surrounding matrix resin. In this respect, slightly aligned and polarized CNTs under AC electric field may locally have profound effects upon entanglements of polymer chains during free radical polymerization. This affects the distribution of charged free radicals generated by decomposition of initiators within the resin. In other words, since they are charged particles, distribution of free radicals may vary, depending on whether the resin suspensions are cured with or without application of the AC electric field. In a similar manner, catalyst particles attached to the surface of CNTs may more significantly affect the extent of free radical polymerization under AC electric field.

Fig. 4a and b depict the SEM images acquired at different magnifications with In-Lens and QBSD detectors in variable pressure (VP) mode at $30 \mathrm{kV}$ for the composite containing $0.05 \mathrm{wt} \%$ of MWCNTs cured with application of the AC electric field. Fig. 4a gives the cross section of the sample cut parallel to the AC electric field applied. As seen in the figure, two different phases are apparent on the fracture surface of the sample. Specifically, white spots refer to doped silver particles, while the black ones refer to CNT clusters. Silver particles doped onto fracture surfaces make it possible to acquire successfully the SEM images at $30 \mathrm{kV}$ without any charging problem. When we look closer (at higher magnification), individual carbon nanotubes, as indicated by the arrows in Fig. 4b, are located around the partially aligned clusters. This shows con-

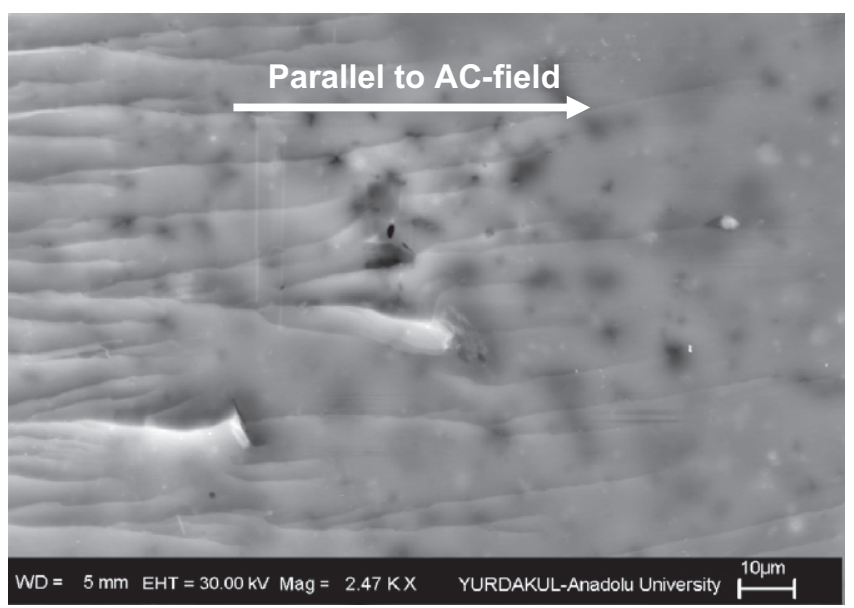

(a)

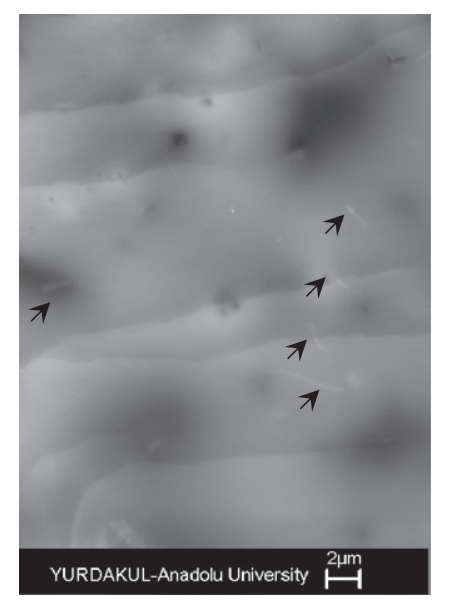

(b)

Fig. 4. SEM micrographs acquired with In-Lens and QBS detectors in VP mode for 0.05 wt.\% of MWCNTs containing samples cured with application of the AC electric field at (a) high and (b) low magnifications. 
sistency with the findings obtained from light microscopy. Indeed, the processing parameters, such as resin viscosity and the shear rate applied during dispersion process do play a pivotal role in the characteristic of CNT agglomerates that govern the conductivity of the resulting composites. In brief, the shear induced by 3-roll milling enables proper dispersion of CNTs within the resin. The increased number of individual CNTs and relatively small size of agglomerates allow the formation of electric anisotropy in the composites cured with the application of the AC electric field.

Fig. $5 \mathrm{a}$ and $\mathrm{b}$ are bright field (BF) TEM images showing the achieved dispersion state of MWCNTs, at 0.05 wt.\% loading rate cured with and without application of the AC electric field, respectively. Fig. 5a shows AC electric field induced aligned individual CNTs, indicated with arrows, while Fig. 5b depicts homogenous dispersion of CNTs in the polymer without any significant alignment. In fact, it is a big challenge to find a well aligned portion in the polymer via TEM. However, it can be said that these observations show consistency with those obtained via SEM examination. Light microscopy findings are also highly proportional to those obtained via TEM. The metallic impurities (Fe, Co) may hold vital clues to the formation of electric anisotropy in the compos-

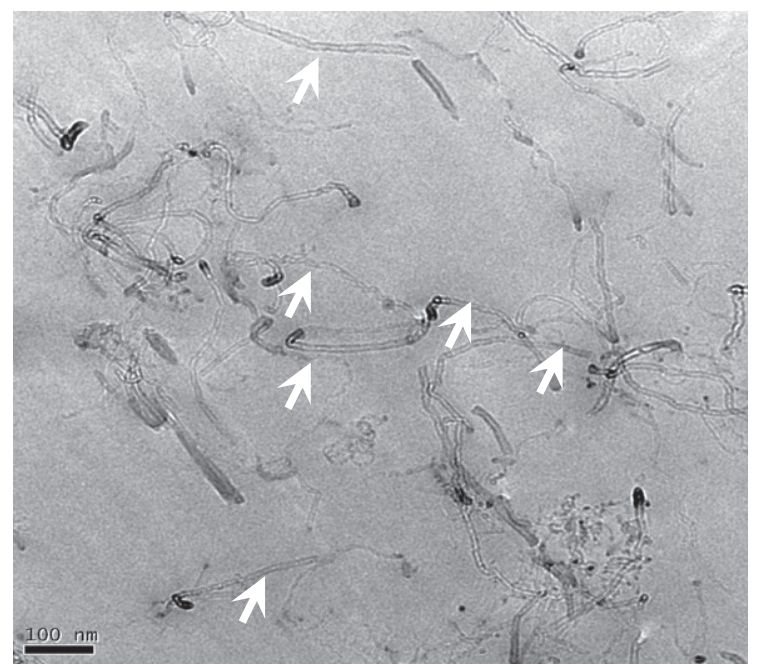

(a)

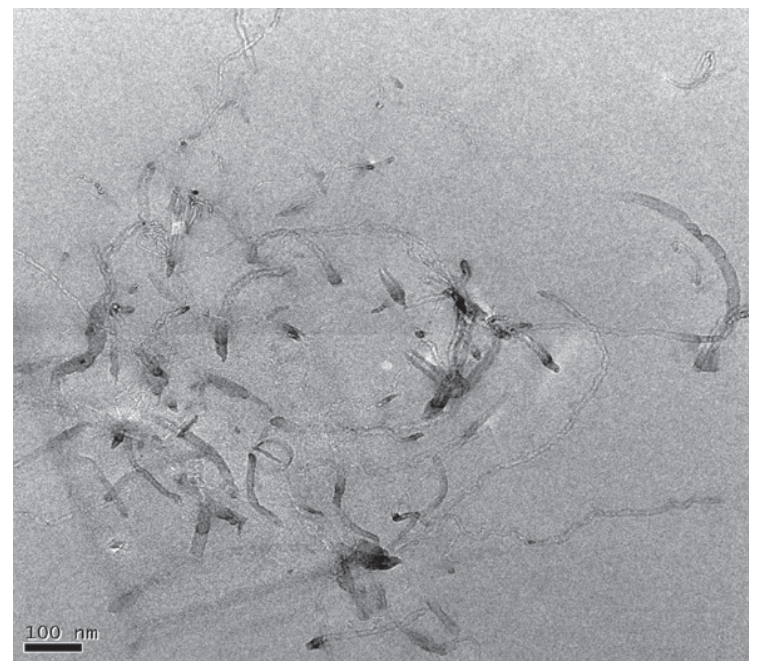

(b)

Fig. 5. Bright field (BF) TEM images showing the achieved dispersion state of MWCNTs, at 0.05 wt.\% of MWCNTs, cured (a) with and (b) without application of the AC electric field. ites. In addition, the chemical nature of impurities may be crucial to the extent of free radical polymerization under AC electric field. TEM equipped with a HAADF detector was in this manner conducted to further characterize the MWCNTs in the same sample $[15,16]$. This technique allows images to be acquired thanks to collection of high angle-scattered electrons. Unlike conventional TEM dark field (DF) images obtained from the signal emitted by elastic scattering of electrons at low angles, STEM-HAADF images emerge from the signal emitted by inelastic scattering of electrons at relatively high angles [16]. In this respect, this technique enables to simply distinguish the regions of different atomic numbers in the CNT modified polymer composites. Elastic and inelastic interactions between the high angle sensitive atoms in the sample and the incident electrons constitute the contrast imaging. Since inelastic scattering is dependent on the number of electrons, the scattering intensity varies with the atomic number.

Fig. $6 a$ and $b$ give STEM-HAADF images of MWCNTs in the composites cured without and with application of the AC electric field, respectively. As seen in Fig. 6a, without electrical field, indefinite compound with high atomic numbers relative to carbon (C) was found attached to the outer surface of MWCNTs, as indicated by an arrow in the micrograph at higher magnification. Interestingly, as seen in Fig. 6b, no similar indefinite particles, but oxidized ends of carbon nanotubes have bright appearance over the surface of MWCNTs in the polymer cured with application of the AC electric field. The reason that CNTs appear brighter than usual during STEM-HAADF examination is due to the fact that oxygen has a higher atomic number than carbon. Generally speaking, the brighter regions apparent in a STEM-HAADF image, the higher atomic number these corresponding regions have. In this respect, our assumption is that catalyst residues left over the surface of CNTs are capable of moving across the part under AC electric field, which in turn has a significant impact on the final properties of the resultant composites. To verify our assumption, EDX elemental analysis was then carried out on the STEM-HAADF images acquired. Fig. 7 gives the point EDX spectrum obtained from the white spots in STEM operating mode apparent on these corresponding images. $\mathrm{Fe}, \mathrm{Co}, \mathrm{C}$ and $\mathrm{O}$ elements were eventually predicted to exist in the chemical compositions of the analyzed region, which is entirely consistent with our assumption as well. To further prove this approach, the distribution of elements indicated by point EDX elemental analysis was justified at higher intensity values via EDX elemental mapping on the same STEM-HAADF image acquired. Fig. 8a-d give EDX elemental mapping images obtained by characteristic X-ray lines, C-K (284 eV), O-K (532 eV), Fe-K (6400 eV) and Co-K $(7100 \mathrm{eV})$, correspondingly. Based on the maps, it is definitely certain that white spots apparent on the outer surface of MWCNTs (given in Fig. 6a) are comprised of only Fe and Co elements. Very small peak of $\mathrm{O}$ refers to oxidized end of untreated CNTs, as highlighted in Fig. 8b. This finding is highly consistent with our approach. As elucidated earlier, the metallic impurities Fe and Co can be relevantly used as an efficient tool to identify the extension of CNT alignment induced by the electric field in the polymer.

The detailed knowledge of elemental composition on the nanoscale is therefore needed for Fe and Co particles attached to the surface of CNTs. As seen in Fig. 6b, no regions brighter than the indefinite particle apparent in Fig. 6a are noticeable on the image, which means that no Fe or Co particles are attached to the surfaces of CNTs. Note that edge effects emerge from the thickness variation and look like a reflection from the surface of CNTs. In the most probable scenario, Fe and Co particles on the surface of CNTs may have changed their locations due to the application of the AC electric field. This may directly affect the extent and characteristics of radical polymerization taking place in the system. Similarly, free radicals generated by decomposition of the initiator are charged particles and capable of moving in the liquid medium 

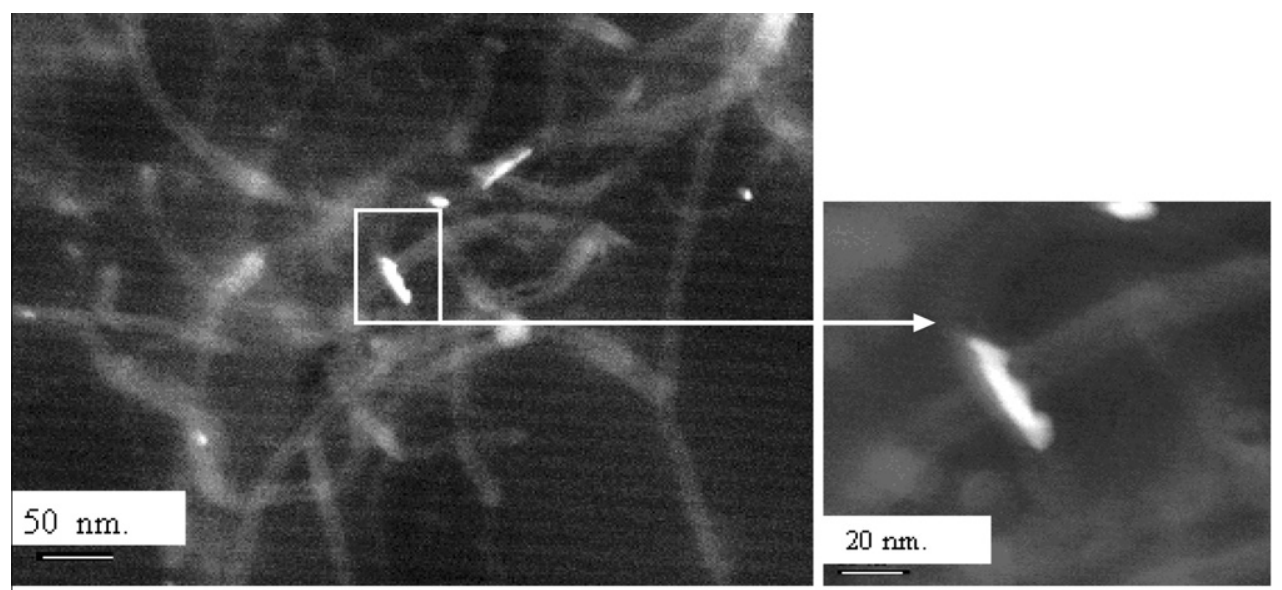

(a)
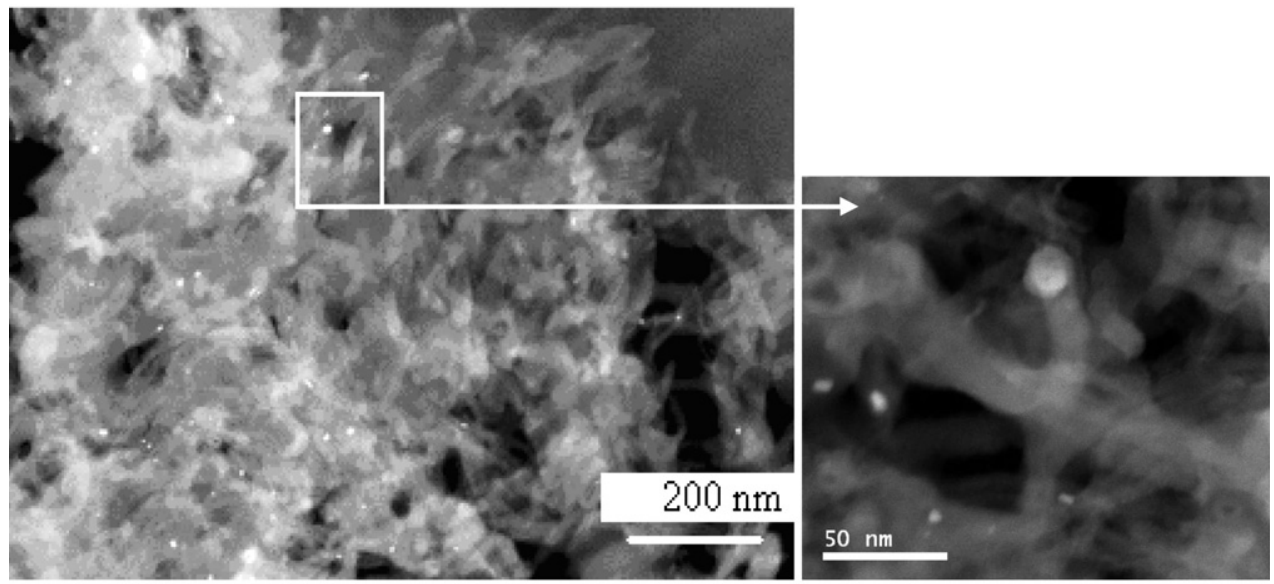

(b)

Fig. 6. STEM-HAADF images of MWCNTs in the composites cured (a) without and (b) with application of the AC electric field.

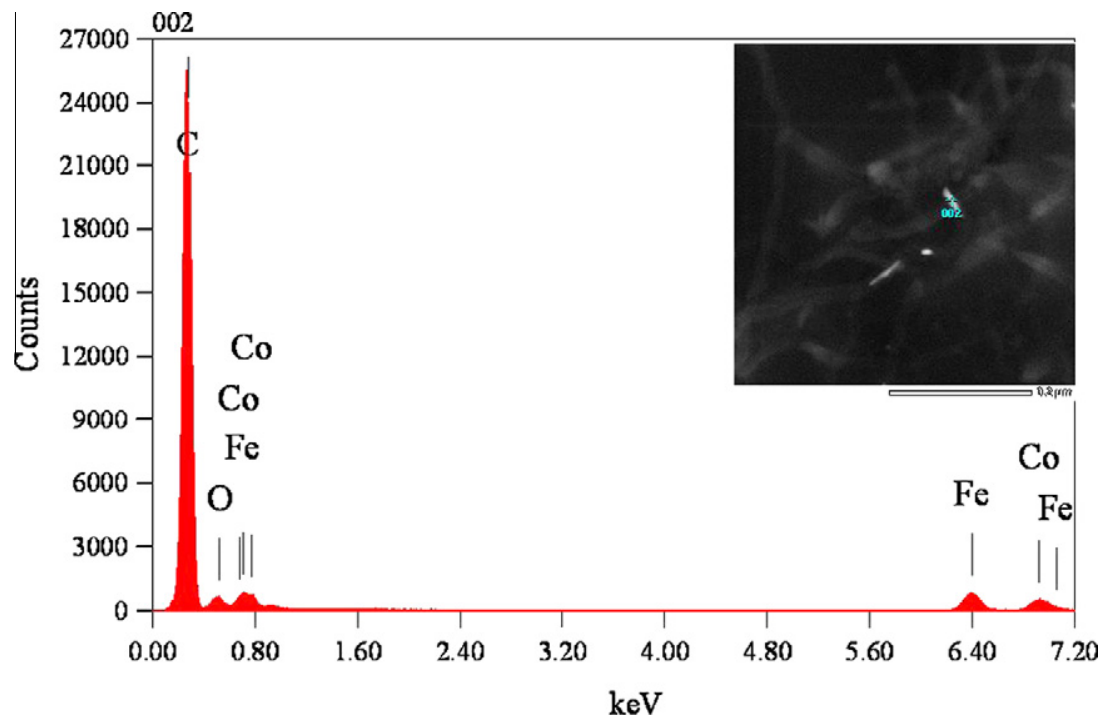

Fig. 7. The point EDX spectrum obtained from the white spots in STEM operating mode apparent on the corresponding images.

under the electric field, which is directly associated with the crosslinking density of the final product. Akbulut et al. [17] investigated the effects of an applied electric field of $1-3 \mathrm{kV} / \mathrm{cm}$ on the polymer- ization of $\alpha$-methyl styrene. They found that the initial rate of monomer disappearance is the same regardless of the absence or presence of the electric field, and that the rate of polymer forma- 


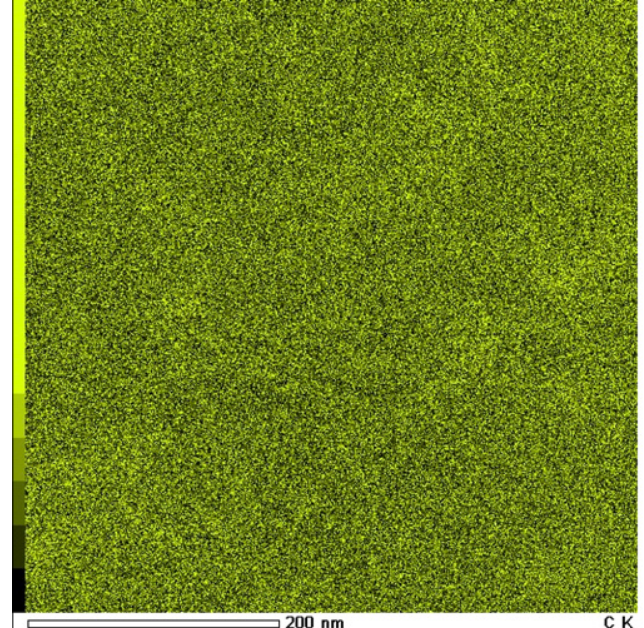

(a)

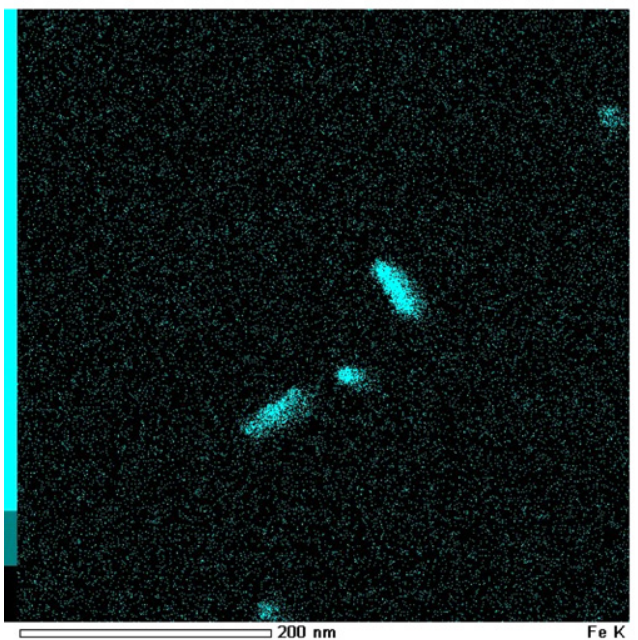

(c)

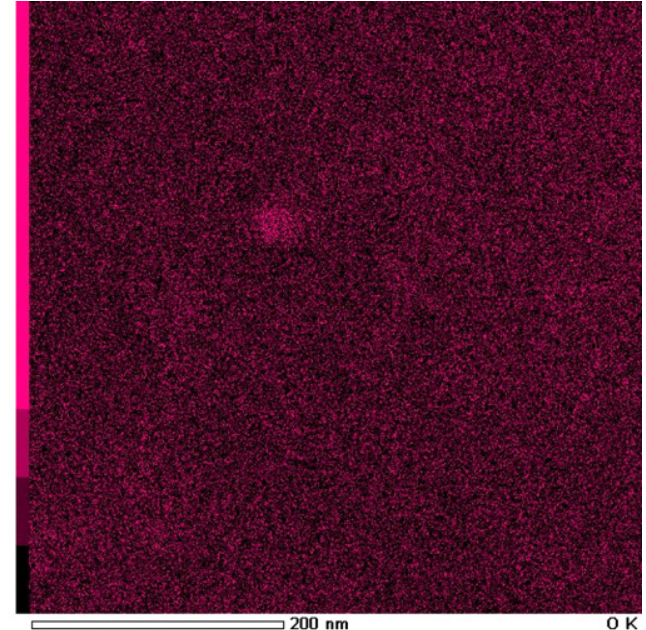

(b)

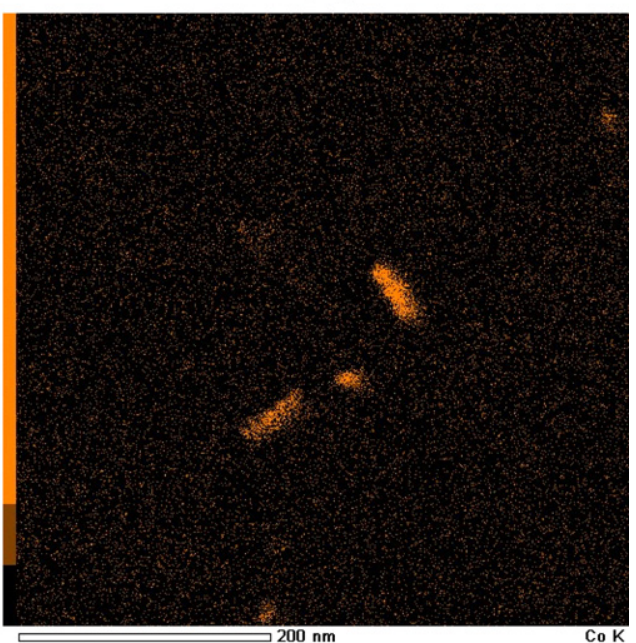

(d)

Fig. 8. EDX elemental mapping images obtained by characteristic X-ray lines, (a) C-K (284 eV), (b) O-K (532 eV), (c) Fe-K (6400 eV) and (d) Co-K (7100 eV).

tion at high conversions decreases when the electric field is imposed on the system. More interestingly, they observed that the NMR, UV absorption, and fluorescence spectra of the polymers are significantly affected by the electric field applied. Therefore, it is reasonable to investigate the final properties of the composites cured with and without application of the electric field.

Fig. 9a-c depict the measured $T_{\mathrm{g}}$ of the neat polymer and its composites containing $0.05 \mathrm{wt} . \%$ of MWCNTs cured without and with application of the AC electric field, respectively. As a result, it was found that there was almost no significant change in the $T_{\mathrm{g}}$ values between the neat polymer $\left(93^{\circ} \mathrm{C}\right)$ and the composites $\left(95^{\circ} \mathrm{C}\right)$ cured without application of the AC electric field. However, composites cured with application of the AC electric field were observed to exhibit relatively high $T_{\mathrm{g}}$ value $\left(107^{\circ} \mathrm{C}\right)$, which corresponds to an improvement of about $10 \%$ compared to the neat polymer. Gryschuck et al. [18] reported that MWCNTs, dependent on their surface area and aspect ratio, may have some adverse effects on the chemical reactions during free radical polymerization of vinyl ester resin. They emphasized that the amount of accelerator and initiator to be added to the CNT modified resin suspensions need to be optimized. They additionally concluded that nanotubes with higher aspect ratio would be more beneficial to the ultimate performance of brittle polymers such as vinyl ester. Similarly, Peng et al. [19] showed that free radicals generated by the decomposi- tion of initiator (MEKP) are partially trapped within the galleries of CNTs during room temperature cure, which impedes the polymerization reaction to some extent and thus reduces the crosslinking density of the final product. Indeed, free radicals are charged particles that can move across the AC electric field. Detachment of Fe or Co particles from the surface of CNTs due to the AC electric field applied, which is confirmed by TEM examination, may allow the entrapped radicals to come into play towards the end of the polymerization reaction. From this viewpoint, the application of AC electric field during cure of the resin suspensions may alter free radical polymerization to some extent. In the most probable scenario, the radicals trapped within the galleries of CNTs were encouraged by the applied AC electric field strength to move, recombine and terminate with another nearby radical, thus contributing to the ongoing chain polymerization. In other words, thanks to the AC electric field applied, CNTs discontinue to act as inhibitors which come into contact with radicals and convert them to lower reactive species that do not react any more. Therefore, reorientation of larger CNT agglomerates and partial alignment of individual CNTs may have brought a synergy to the resultant composites, just modifying the distribution of radicals in the resin. This is why we obtained a higher $T_{\mathrm{g}}$ from the polymer cured with application of the AC electric field. Schwarz et al. [6] concluded that, in contrast to epoxy resins, the chemically neutral vinyl ester resin 


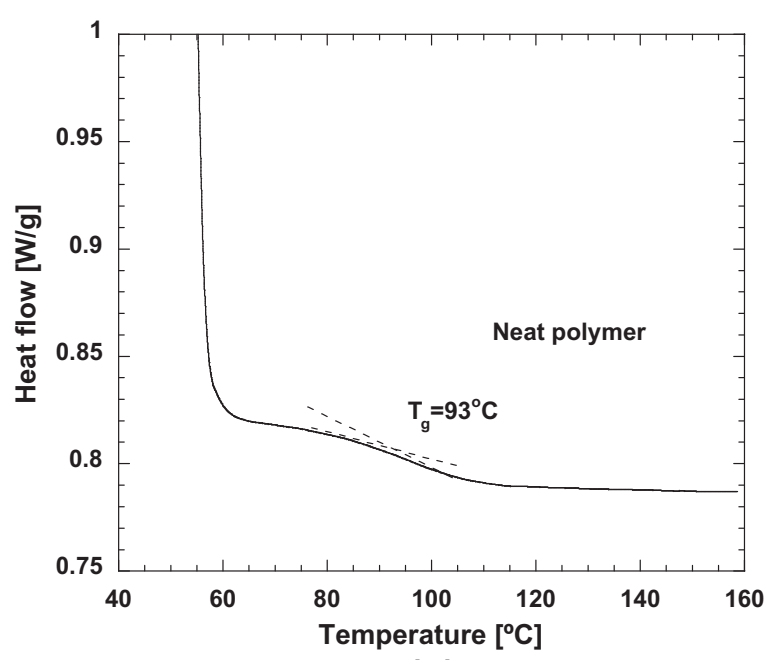

(a)

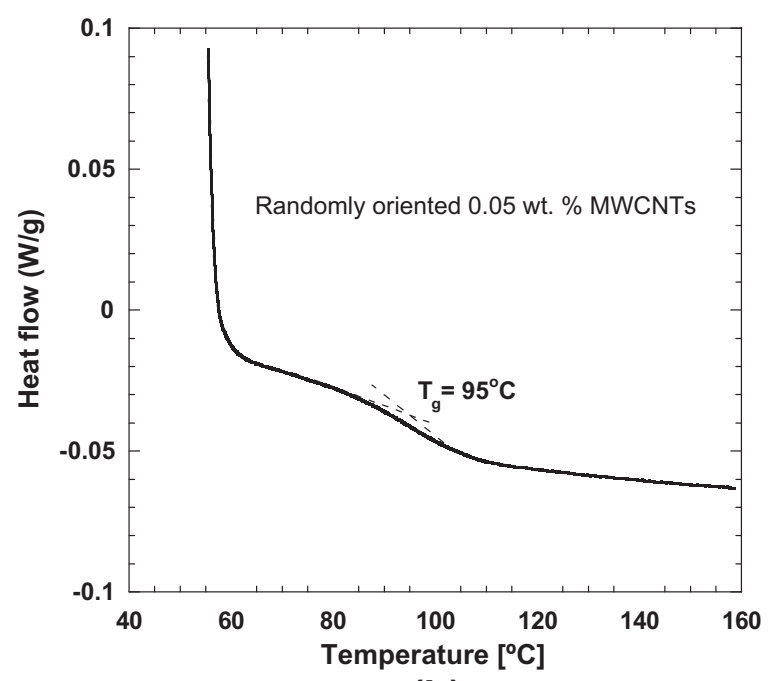

(b)

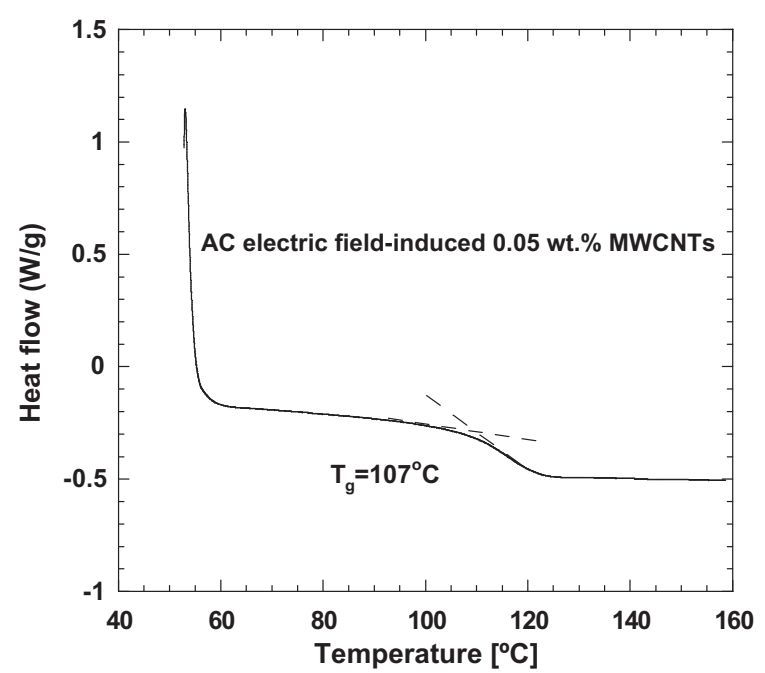

(c)

Fig. 9. The measured $T_{\mathrm{g}}$ values of neat polymer (a) and its composites containing $0.05 \mathrm{wt}$ \% of randomly oriented (b) and AC electric field induced aligned MWCNTs (c).

produces no excess charge on the $\mathrm{CB}$ particles, and that the ionic conductivity of the matrix is low. As a result, regardless of AC or
DC fields, field-induced agglomeration as well as cluster formation occurs in the bulk. Note that a several orders of magnitude higher conductivity value, as stated earlier, was obtained from the polymer cured with application of the AC electric field. In summary, it can be concluded that AC electric field induced CNT agglomeration and cluster formation together with the movement of charged radicals across the field favor the electrical conductivity and the thermal properties of the resulting composites.

\section{Conclusions}

In this study, double and multi-walled carbon nanotubes with and without amine functional groups (DWCNTs, MWCNTs, DWCNT- $\mathrm{NH}_{2}$ and MWCNT- $\mathrm{NH}_{2}$ ) were considered as highly promising conductive fillers in a vinyl ester resin. Low content of carbon nanotubes (CNTs) was dispersed in the matrix resin via 3-roll milling. Regardless the type of CNTs or their content, the resulting polymer composites with amino-functionalized CNTs were found to exhibit lower electrical conductivity values than those with untreated CNTs. More specifically, at each given concentration, the highest and the lowest electrical conductivity values were accomplished in the composites prepared with MWCNTs and DWCNT$\mathrm{NH}_{2}$, respectively. Since MWCNTs showed the highest electrical conductivity, further experiments aimed to induce the formation of aligned conductive CNT networks during cure by the application of an alternating current $(A C)$ electric field were carried out on the resin suspensions with MWCNTs. Electrical conductivity measurements performed on the cured samples verified the formation of electric anisotropy due to the alignment of CNTs within the polymer. Light microscopy, SEM and TEM were then used to visualize the alignment of CNT networks from fracture surfaces of the polymers. Further, it was found that the catalyst particles (Fe and $\mathrm{Co}$ ) on the surface of MWCNTs may disappear across the part during cure, whilst the AC electric field was applied. This makes the difference between the characteristics of the polymer composites cured with and without application of the AC electric field. DSC revealed variations in $T_{\mathrm{g}}$ of the polymers cured with and without application of the AC electric field. Polymers containing 0.05 wt.\% of MWCNTs aligned in the electric field possessed a significantly higher $T_{\mathrm{g}}$ than the neat polymer or polymer containing similar content of MWCNTs cured without application of the AC electric field.

\section{Acknowledgements}

The authors acknowledge TUBITAK of Turkey and BMBF of Germany for the financial support under Project No.: 5 and Poliya Polyester A.S., Istanbul for providing us with the resins used in this study. The authors would also like to thank Dr. Josef Kovacs for his valuable help in the construction of the experimental set up used to align CNTs within the polymer. The authors would also like to thank Prof. Dr. H. Mehtap Kutlu for her fruitful discussion and comments on preparation of TEM thin polymer samples.

\section{References}

[1] Ajayan PM, Charlier JC, Rinzler AG. Carbon nanotubes: from macromolecules to nanotechnology. Science 1999;96:14199-200.

[2] Sandler J, Shaffer MSP, Prasse T, Bauhofer W, Schulte K, Windle AH Development of a dispersion process for carbon nanotubes in an epoxy matrix and the resulting electrical properties. Polymer 1999;40:5967-71.

[3] Sandler JKW, Kirk JE, Kinloch IA, Shaffer MSP, Windle AH. Ultra-low electrical percolation threshold in carbon nanotube-epoxy composites. Polymer 2003;44:5893-9.

[4] Şimşek Y, Özyüzer L, Seyhan AT, Tanoglu M, Schulte K. Temperature dependence of electrical conductivity in double-wall and multi-wall carbon nanotube/polyester nanocomposites. J Mater Sci 2007;32:9689-93.

[5] Munson SH. Estimation of the critical concentration in an anisotropic percolation network. Phys Rev B 1991;43:3331-6. 
[6] Schwartz MK, Bauhofer W, Schulte K. Alternating electric field induced agglomeration of carbon black filled resins. Polymer 2002;43:3079-82.

[7] Celzard A, Mcrae E, Deleuze C, Dufort M, Furdin G, Mareche JF. Critical concentration in percolating systems containing high aspect ratio filler. Phys Rev 1996;53:6209-14.

[8] Prasse T, Cavaille JY, Bauhofer W. Electric anisotropy of carbon nanofibre/ epoxy resin composites due to electric field induced alignment. Compos Sci Technol 2003;63:1835-42.

[9] Prasse T, Flandin L, Schulte K, Bauhofer W. In-situ observation of electric field induced agglomeration of carbon black in epoxy resin. Appl Phys Lett 1998;72:1-3.

[10] Martin CA, Sandler JKW, Windle AH, Schwarz MK, Bauhofer W, Schulte K, et al. Electric field induced aligned multi wall carbon nanotube networks in epoxy composites. Polymer 2005;46:877-86.

[11] Martin CA, Sandler JKW, Shaffer MSP, Schwarz MK, Bauhofer W, Schulte K, et al. Formation of percolating networks in multi wall carbon nanotube-epoxy composites. Compos Sci Technol 2004;64:2309-16.

[12] Kovacs JZ, Andresen K, Pauls JR, Garcia CP, Schossig M, Schulte K, et al. Analyzing the quality of carbon nanotube dispersions in polymers using scanning electron microscopy. Carbon 2007;45:1279-88.
[13] Loos J, Alexeev A, Grossiord N, Koning CE, Regev O. Visualization of single wall nanotube (SWNT) networks in conductive polystyrene nanocomposites by charge contrast imaging. Ultramicroscopy 2005;104:160-7.

[14] Gojny FH, Wichmann MHG, Köpke U, Fiedler B, Schulte K. Carbon nanotube reinforced epoxy composites - improved (fracture) mechanical properties at low nanotube contents. Composites Part A 2004:2363-71.

[15] Friedrich H, McCartney MR, Buseck PR. Comparison of intensity distributions in tomograms from BF TEM, ADF STEM, HAADF STEM, and calculated tilt series. Ultramicroscopy 2005;106:18-27.

[16] Akita T, Tanaka K, Okuma K, Koyanagi T, Haruta M. TEM and HAADF-STEM study of a $\mathrm{Au}$ catalyst supported on a $\mathrm{TiO}_{2}$ nano-rod. J Electron Microsc 2001;50:473-7.

[17] Akbulut U, Birke RL, Fernandez JE. Electric field effects in the polymerization of $\alpha$-methylstyrene. J Polym Sci 2003:2507-13.

[18] Gryschuck O, Kocsis JK, Thomann R, Kanya Z, Kiricsi I. Multiwall carbon nanotube modified vinyl ester and vinyl ester-based hybrid resins. Composites Part A 2006;37:1252-61

[19] Peng H, Reverdy P, Khabashesku VN, Margrave JL. Sidewall functionalization of single walled carbon nanotubes with organic peroxides. J Am Chem Soc 2003;125:15174-84 